\title{
The design of data analysis system for user
}

\author{
Jiawei Shen ${ }^{1, a^{*}}$, Yawen $\mathrm{Dai}^{2, \mathrm{~b}^{*}}$ and Zhencai $\mathrm{Li}^{3, \mathrm{c}}$ \\ ${ }^{1}$ Wuhan University of Technology, China \\ a1069870257@qq.com, bdaiyaweny@163.com, '627787952@qq.com
}

Keyword: LabVIEW; data analysis; data unpacked; Fourier transform; FFT; DFT;

\begin{abstract}
In communications engineering, we often encounter some problems of the data analysis and processing aspects, therefore analysis of engineering data throughout the project has become a crucial part. LabVIEW is a program development environment, it is developed by National Instruments (NI) company developed, it is similar to C and BASIC development environment [1]. However, a significant difference between LabVIEW and other computer languages are: other computer languages are based on test code, and the use of LabVIEW graphical programming editor language $\mathrm{G}$, the program is produced in block diagram form. LabVIEW software is the core design platform of NI company, is an ideal choice for the development of measurement or control system. LabVIEW development environment allows engineers and scientists to have all the necessary tools to quickly build a variety of applications [2]. LabVIEW development environment is designed to help engineers and scientists to solve problems and improve productivity and innovation. This paper will introduces a LabVIEW program design for data analysis in detail.
\end{abstract}

\section{Introduction}

In some of the data acquisition and monitoring system, it is often need to analyse the data we have collected, that made it easy for users to clearly understand the situation of engineering, so that we can know in time when problems happened, so as to quickly solve the problem. This paper presented how we can make an analysis of the real-time data by LabVIEW in an engineer project, to solve this problem, it makes us easily get the analysis of the data in time domain and frequency domain.

The LabVIEW as a development with strong practicability, can accurately simulates experiment instruments, and even many experimental instrument processing is not the problem, so it often plays a key role in engineer programs. The virtual experiment platform has good portability and safety reliability, it allows multi-users and multi-experiments to be carried out at the same time. This article describes the platform of the LabVIEW with related functions and how we programed to achieve related functions such as data analysis.

\section{File I/O application in LabVIEW}

In the process of using the LabVIEW program, often need us to store the data or to read data, so we need the file I/O application. LabVIEW offers for multiple file types in the format of the "data $\mathrm{read} /$ write” operation function, used to realize data storage and reading. When using LabVIEW for measurement and detection, often need to store different types of real-time test data, so that we can make data analysis, replay waveform and generate various types of reports. LabVIEW provides a rich file type to meet users' demand for storage format [3]. There are 8 kinds common file types, this experiment used a text document to store the data. 
LabVIEW provides two ways to create a text file. One way is to use the "open/create/replace” files function, the other easier method is to use written function. Here we have already put the data into TXT text documents; now what we need to do is to read the text document data. To use the function we must set the related parameters first. This function has some interface, here is what we need to set are file path interface, operating interface and permissions interface. Set file path parameter to the file path where you need to read, set operate parameter to open, permissions set to read.

And then we need to set some the relevant parameters of Read from Binary File function. Because of the need to read the text of the document data quantity is large, if we read all the data at a time will cause the program to death, so here we'd better read only a certain number of bytes of data at a time, you need to set total data parameter to 1000, representing every time read 1000 bytes of data from the text document. At the same time connect file (using the dialog box) connect with output file of “open/create/replace” function.

After reading the text document, you must close the text documents to read, so as to ensure the integrity of the program. Usually we use the close file function.

In order to ensure the program running in high-speed and effectively, we can put open file and close file out of the while loop execution, if we put them inside the while loop, it will waste a lot of time to execute these step, that would reduce the efficiency of program and take a great occupancy rate of CPU.

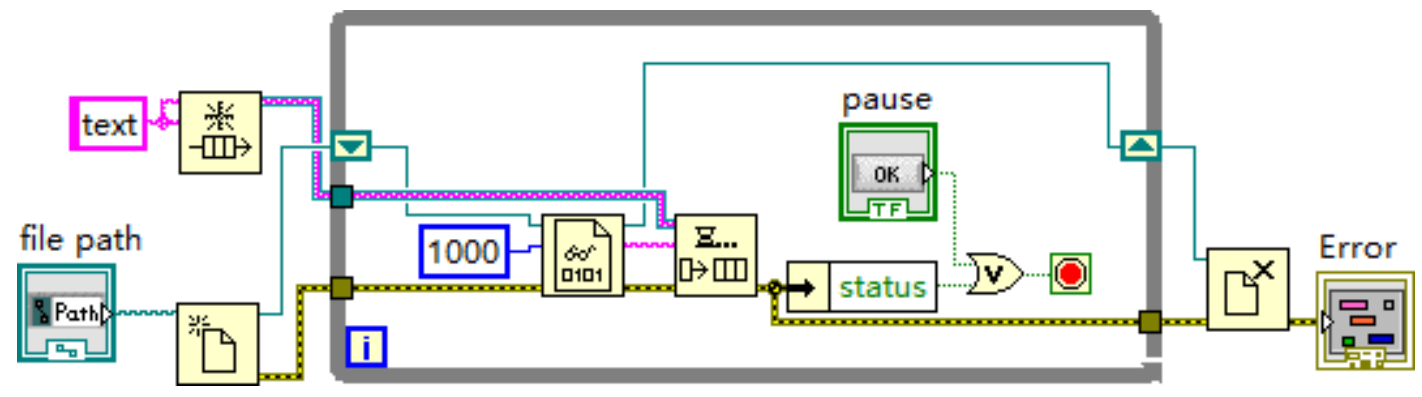

Fig. 1. The program of text file reading

\section{Data processing}

LabVIEW developers provided us with many modules, a variety of data types can be convert to each other through these function. After reading the text document, the data types are string, if we want to make a mathematical analysis of the data, data must be converted to the corresponding data types such as floating point. In addition, we still need to unpack the 1000 bytes strings for the correct data formats. 


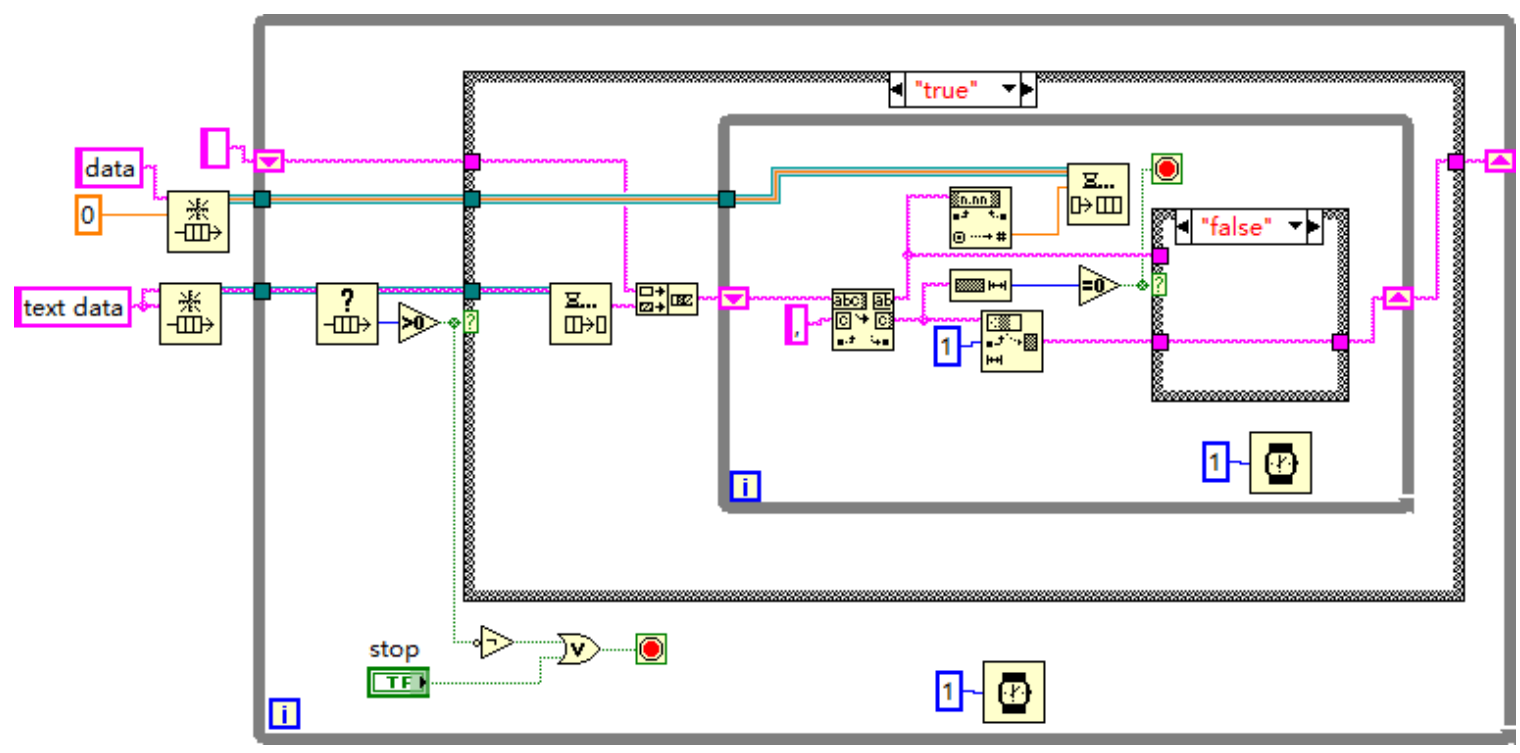

Fig. 2. The program of data unpacking

Developers to provide us with the waveform chart controls, it can save our program development time, and this control can perfectly simulate a real oscilloscope. Unlike real oscilloscope and virtual instrument ordinate represents the size of the data waveform chart, the abscissa represents sample point sequence. Like oscilloscope used in the experiment, LabVIEW waveform graphs can also range the waveform data area, choose specific area and enlarge the scope of the waveform processing.

In this paper, the data analysis is the selection of data for the comparison of the numerical size selection of the maximum and minimum values. The user can select the particular range of data, then click on the "data analysis" button data will be the segment and you can get the spectrum.

This experiment will read the string data from a text document then convert to numerical data, the data will be output in form of queue at regular intervals shown in the waveform chart. When using waveform chart we need to set several parameter, abscissa fixed sampling points, keep the length of historical data in an appropriate size. These operations require the use of attribute node this special controls. Here we initialize the waveform chart, setting the waveform chart the Maximum of X axis to 3000 , the waveform chat history to an empty array. Innovation point of this experiment is to select the area on waveform chart first then make analysis of the part selected.

Waveform chart have a zoom control, using zoom control select an area on the waveform chart, after scaling Maximum and minimum values of $\mathrm{X}$ axis will be changed. The serial number of the data to be analyzed is between these two values, the other part will be abandoned.

After the selected to analyze the data range, the next thing to do is analyzing the part of data. Here we set up a "data analysis" button as a Boolean control on the front panel, the users can click on the button to complete the data analysis. So we must use event cases structure in LabVIEW to complete the function of data analysis. Setting “data analysis" button as pending events cases, wait for the program to respond it. Put the event structure inside the while loop, and set the timeout for $100 \mathrm{~ms}$, program will continuously detect the occurrence of the event case.

In the other while loop, program will do the output queue data analysis processing. 


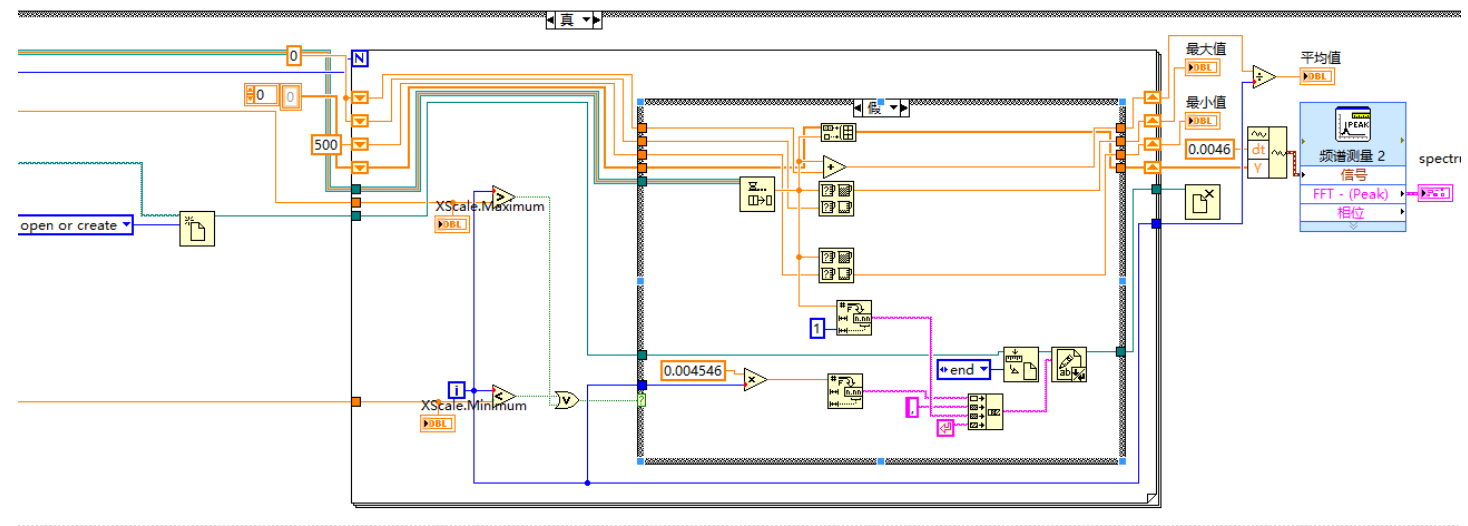

Fig. 3. The program of data processing

The while loop in the LabVIEW environment greatly limits the freedom of data transmission, but the developers can use to a local variable data calls, or transfer data in the form of a queue to the interior of the while loop.

\section{Data analysis}

This experiment chose to put the data to be processed in the form of a queue to enter inside the while loop, the queue has an attribute of FIFO, so that we can guarantee the data collected inside the while loop continuous uninterrupted for data analysis.

In addition to the above mentioned to display the basic data, calculate a selected range of maximum, minimum, average, the data within the scope of this experiment is to select the frequency spectrum analysis. This experiment used spectrum measurement function for spectrum analysis of the data. After entering data, first of all, we used fast Fourier transform with FFT, made the data signal quickly separated into an infinite numbers of synthetic signal by sine and complex exponential signal, made the signal in form of the sum of sine signal, and make it expansion according to the frequency, become a function of frequency, then study and processing the signal in the frequency domain [4].

For one dimensional signal, the VI fast Fourier transform algorithm is used to calculate the input sequence of discrete Fourier transform (DFT). One dimension DFT is defined as:

$$
Y_{k}=\sum_{n=0}^{N-1} X n e^{-2 \pi k n / N}, n=0,1,2, \ldots, N .
$$

$X$ is the input sequence, $N$ is the number of elements in $X, Y$ is the result of a transformation. The frequency resolution of $Y$ 's component (frequency space) is:

$$
\Delta f=\frac{f s}{N}
$$

$\mathrm{f}_{\mathrm{s}}$ stands for sample frequency.

Function of $x(t)$ power spectrum $S_{x x}(f)$ is defined as:

$$
\begin{aligned}
& S_{x x}(f)=X^{*}(f) X(f)=|X(f)|^{2} . \\
& X(f)=F\{x(t)\} .
\end{aligned}
$$

$X^{*}(f)$ is a complex conjugate of $X(f)$. This VI can compute power spectrum based on FFT and DFT [5]. 
$S_{X X}=\frac{|F\{X\}|^{2}}{n^{2}}, S_{X X}$ stands for the power spectrum of the output sequence, $\mathrm{n}$ stands for sampling number of input sequence.

When the sample number $n$ of input sequence $X$ is integer power of $2, n=2^{m}, m=1,2,3, \ldots, 23$, through rapid FFT algorithm based on 2 calculating numerical sequence of fast Fourier transform and scale square amplitude.

Sampling number is not valid in the input sequence $X$ is a power of 2, but the decomposable factor of small prime product, through efficient numerical sequence DFT algorithm is real discrete Fourier transform and scale square amplitude, through fast DFT can calculate maximum power spectrum $2^{22}-1$. Assumption $Y$ is the Fourier transform of the input sequence $X, \mathrm{n}$ is sampling number in the input sequence, so that we can get

$$
|Y n-j|^{2}=|Y-j|^{2} \text {. }
$$

The power of the (n-1)th element in $Y$ can be understood as a sequence of the i-th element of power. Sequence represents the ith harmonic power. By the following equation we can get the ith harmonic (not including DC component and Nyquist component) of the total power [6].

The power of ith harmonic wave is equal to

$$
2\left|Y_{i}\right|^{2}=\left|Y_{i}\right|^{2}+\left|Y_{n}-1\right|^{2}, \quad 0<i<n / 2
$$

The total power of the DC component and Nyquist components is respectively $\left|Y_{0}\right| 2$ and $\left|Y_{n} / 2\right| 2$. Test results as follows:

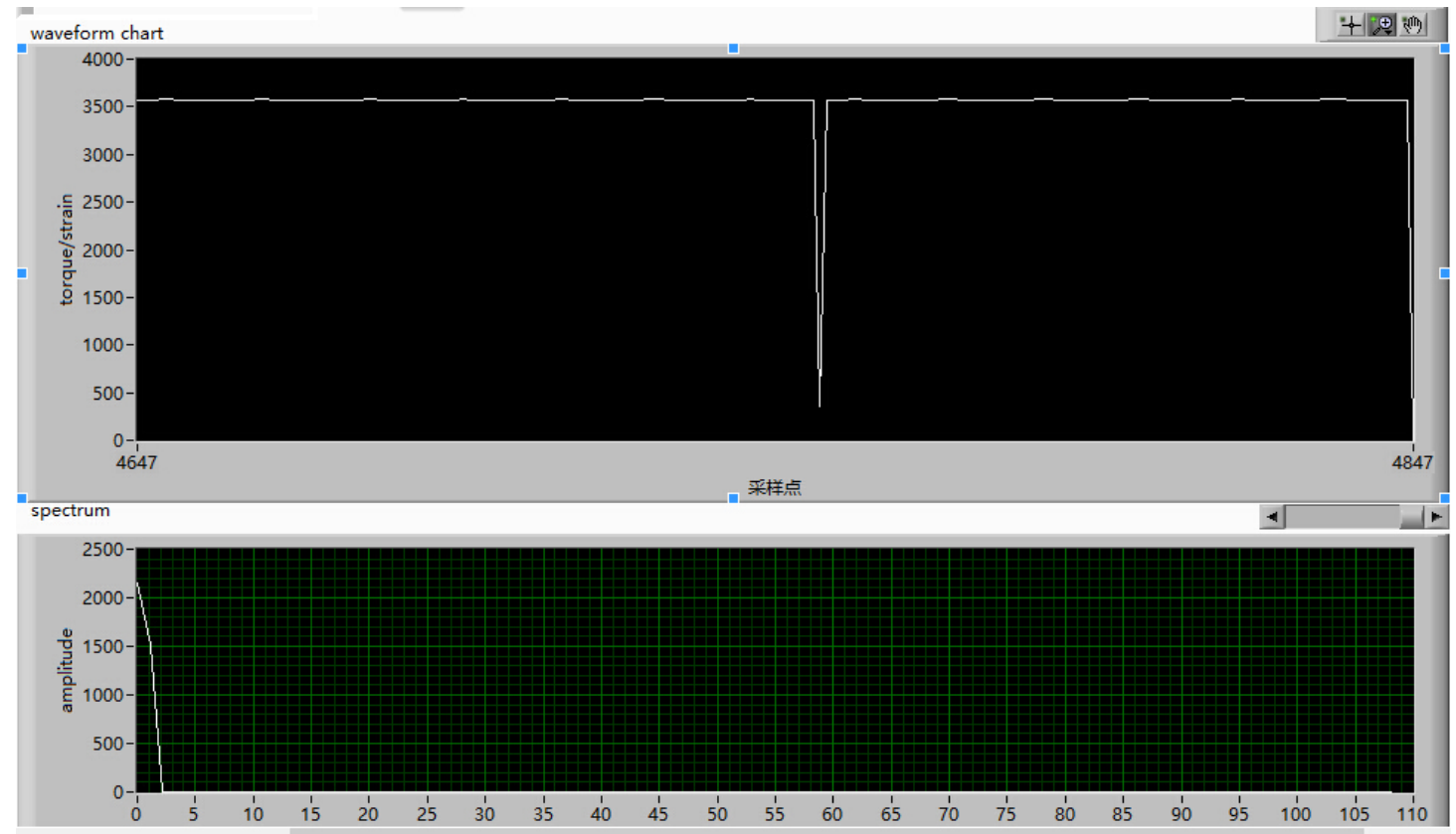

Fig. 4. The test results

The LabVIEW program is not only has friendly interface, simple operation, convenient debugging, but also has strong expansibility and portability, the generated executable EXE file, can be installed perform in the system absence of the LabVIEW environment.

\section{Conclusion}

In this paper, the experimental platform achieved the function of reading and analyzing the data in a text file by LabVIEW environment. After implementing these basic functions, we can also achieve to further extension of the platform in the future, such as improving the efficiency of software 
implementation, to reduce the occupancy rate of CPU, perfecting the system of more functions, improve the users' interface, so as to further perfect this system step by step.

\section{References}

[1] Sriharsha Vathsavayi, Hadaytullah, Kai Koskimies. Interleaving human and search-based software architecture design[J]. Proceedings of the Estonian Academy of Sciences, 2013, 62(1).

[2] Bax Leon, Yu Ly-Mee, Ikeda Noriaki et al.. A systematic comparison of software dedicated to meta-analysis of causal studies[J]. BMC Medical Research Methodology, 2007, 7(1).

[3] Kruijshaar Michelle, Essink-Bot Marie-Louise, Donkers Bas et al.. A labelled discrete choice experiment adds realism to the choices presented: preferences for surveillance tests for Barrett esophagus[J]. BMC Medical Research Methodology, 2009, 9(1).

[4] $\mathrm{H}$ Ichinose. Crystal interface and high-resolution electron microscopy-the best partner[J]. Science and Technology of Advanced Materials, 2000, 1(1).

[5] Muin Michael, Fontelo Paul, Liu Fang et al.. SLIM: an alternative Web interface for MEDLINE/PubMed searches - a preliminary study[J]. BMC Medical Informatics and Decision Making, 2005, 5(1).

[6] Gong Sungsam, Park Changbum, Choi Hansol et al.. A protein domain interaction interface database: Inter Pare[J]. BMC Bioinformatics, 2005, 6(1). 